\title{
Mindfulness In Action And Time:An Analysis Of 911 Response On September 11, 2001
}

Maria Laura Frigotto and Alessandro Narduzzo

Published Online: 30 Nov 2017 |https://doi.org/10.5465/AMBPP.2017.16915abstract

\section{Abstract}

Research on mindfulness has sought to explain how people respond to the challenge of unexpected events by searching for behaviors and structures that support anticipation and response. In the course of this effort, mindfulness has been defined mainly in terms of static properties, or at most organized in a linear logical sequence. We propose a re-grounding of mindfulness as a dynamic construct that acknowledges the temporal interconnectedness of scanning, interpretation, and response. As an approach to inductively generating theory on mindfulness, we analyzed 911 emergency calls placed during the $102 \mathrm{~min}$ (from the first plane crash to the collapse of the second tower) of the September 11, 2001 terrorist attack in New York. We conceptualize our findings into the Dynamic Model of Mindfulness in Action, adopting causal loop diagrams. This model grounds mindfulness in time and interaction and considers the elapsing in time: the ordering of actions, their duration, pace, interconnectedness, and compatibility with the decision-making time available. We further contribute to existing research by theorizing the role of emotions within the dynamics of mindfulness, showing that they affect the pace, the magnitude, and even the structure of the causal loops of mindfulness in action. 


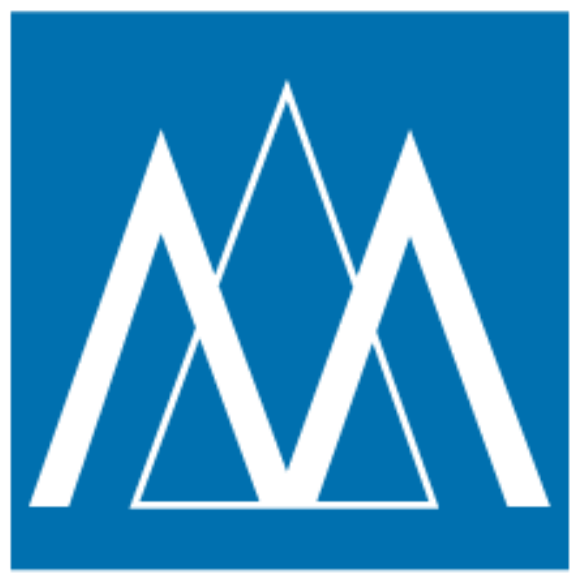

A C A D E M Y O F

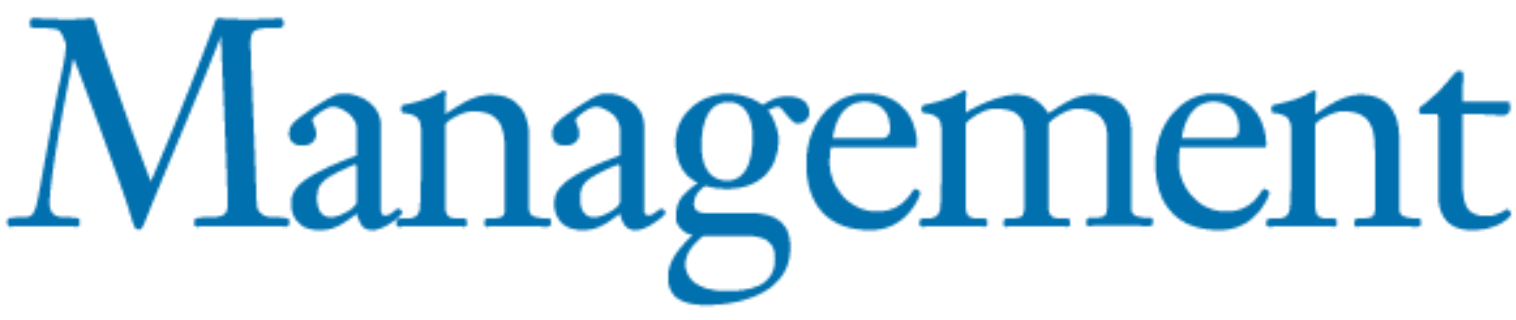

Academy of Management

555 Pleasantville Road, Suite N200

Briarcliff Manor, NY 10510-8020, USA

Phone: +1 (914) 326-1800

Fax: +1 (914) 326-1900

Privacy Policy Logo Use

(c) 2020 Academy of Management

Powered by Atypon ${ }^{\circledR}$ Literatum 